\title{
A Case of Right Posterior Semicircular Canal Benign Paroxysmal Positional Vertigo with to-and-fro Canal Switch to Right Horizontal Semicircular Canal
}

\author{
Ajay Kumar Vats ${ }^{1}$ \\ ${ }^{1}$ Department of Neurology, Chaudhary Hospital \& Medical Research \\ Centre Private Limited, Udaipur, Rajasthan, India
}

Address for correspondence Ajay Kumar Vats, MBBS, MD, DM, Department of Neurology, Chaudhary Hospital \& Medical Research Centre Private Limited, 472-473, Sector 4, Hiran Magri, Udaipur 313002, Rajasthan, India (e-mail: vatsneuro@gmail.com).

Ann Otol Neurotol:2020;3:27-34

\begin{abstract}
Keywords

- canal switch

- canalolithiasis

- Epley's maneuver

- supine roll test

- canalith repositioning maneuver

- positional nystagmus
\end{abstract}

Background Canal switch in BPPV is a phenomenon occurring after therapeutic canalith repositioning maneuvers (CRM), when there is a reflux of the repositioned otoconial debris from utricle to semicircular canal other than the one originally affected. It may be of immediate-type occurring within minutes after CRM or a delayed-type occurring after 2-3 days.

Aim The study is a case report.

Case Report A 59-year-old female presented with history of severe rotational vertigo as she got up from the bed in the early morning at 5.00 a.m.. Dix-Hallpike test (DHT) on the right elicited an upbeating positional nystagmus (PN). Treatment with multiple consecutive modified right Epley maneuvers ( $r$-MEM) in one session was undertaken. During these maneuvers she continued to have an upbeating PN during the 45-degrees right cervical rotation with the neck in 20-degrees of extension (which is equivalent to right Dix-Hallpike positioning) of the first three sequential r-MEM's. With the neck maintained in 20-degrees of extension, during fourth consecutive $r$-MEM, the 45-degrees cervical rotation to right elicited apogeotropic horizontal PN. Supine roll test (SRT) was immediately undertaken. Maximal head yaw positioning to right as well as to the left elicited apogeotropic horizontal PN without torsional component lasting more than one minute, indicating reflux of otoconia from the right P-SCC to the short anterior arm of right H-SCC. She was treated with two sequences of Appiani maneuver and SRT one hour later elicited geotropic upbeating PN with vertigo, indicating second canal switch to P-SCC. It was successfully treated with two sequences of right EM fifteen minutes apart, with instructions to stay upright in between and after the maneuvers. At 24 hours, repeat DHT and SRT were negative and patient was asymptomatic.

Conclusion An optimal time delay to perform a verifying positional test after therapeutic session with CRM is crucial to prevent the immediate type of reflux of relocated otoconia from the utricle into a different semicircular canal. In centers, where more than a single EM is performed in a single session of treatment, a delay of 10 to 15 minutes appears to be appropriate between successive maneuvers. A certain period of restraint in the vertical position after CRM may prevent immediate reflux, but this needs to be confirmed by the randomized control trials.
License terms

() (1) $\Theta \circledast$ 


\section{Introduction}

Benign paroxysmal positional vertigo (BPPV) is a mechanical peripheral vestibular disorder characterized by episodes of rotatory vertigo provoked by head movements that occur relative to gravity. - Table 1 indicates that 56 to $93 \%$ of all BPPV patients diagnosed at any specialty clinic have posterior semicircular canal benign paroxysmal positional vertigo (P-SCC-BPPV)..$^{1-9}$ Evidently, P-SCC-BPPV is the most common variant of all BPPV variants. The abundance of P-SCC-BPPV is attributed to its peculiar anatomy, allowing degenerative otoconial debris derived from utricular macula to readily enter it under the effect of gravitational force.

Typical situations during which episodes of BPPV occur are lying supine, getting up from a supine to sitting position, taking lateral recumbent positions, bending forward to tie shoelaces, and pitching the head up to place an object on a high shelf. Vertigo typically lasts less than 1 minute and episodes longer than 1 minute are considered atypical, raising a possibility of alternative or additional diagnosis. ${ }^{10}$ The pathophysiology consists of inappropriate detachment of otoconial debris from the utricular macula and the debris' entry into any one or more of the SCC, which is called canalolithiasis, or adhering to the cupula, which is called cupulolithiasis. Canalolithiasis is more amenable to treatment with mechanical head maneuvers that aim at repositioning the otoconia from the SCC (where they have inappropriately entered) through the utricular exit in the nonampullary arm of the SCC to the utricle (where they normally remain as a part of utricular the gelatinous matrix). These maneuvers are called canalith repositioning maneuvers (CRMs). CRMs have been developed by various researchers for every SCC by conceptualizing a three-dimensional model of the labyrinth, keeping in mind the ostensible location of the otoconia in the SCC after lateralization and localization of the involved canal by observing the patterns of nystagmus elicited on the diagnostic positional tests. The head along with body of the patient diagnosed with BPPV are sequentially oriented in such a manner that the otoconial debris is moved from its ostensible location within the involved SCC under the gravitational force toward the utricle, keeping in mind that the utricular exit of the canal is located in the nonampullary arm of the SCC.

The phenomenon of reflux of otoconia after CRM into a canal different from the one originally affected is known as canal conversion or canal switch, and such form of canalolithiasis as canal switch BPPV or canal reentry BPPV. In 1996, it was first observed that 85 patients who were diagnosed P-SCC-BPPV and underwent a therapeutic modified CRM; five (6\%) developed anterior semicircular canal BPPV (A-SCC-BPPV) and three (3.5\%) horizontal semicircular canal BPPV (H-SCC-BPPV) during 1 week of follow-up. ${ }^{11}$ The incidence of canal switch from posterior semicircular canalolithiasis to horizontal semicircular canalolithiasis is reported to be 6 to $8 \% 12,13$ and for the other way around it is approximately $6 \% .{ }^{14}$ Canal switch between the anterior and posterior semicircular canalolithiasis has been observed in $2.9 \%$ of patients undergoing CRM. ${ }^{15}$ Conversion of anterior semicircular canalolithiasis to posterior or horizontal canalolithiasis or to horizontal canal cupulolithiasis, either spontaneously or during positional maneuvers, is strongly supportive of the diagnosis of the former. ${ }^{10}$ Canal switch has been classified as immediate type, occurring within minutes after the CRM or a delayed type, occurring after 1 or 2 days after the CRM. ${ }^{16}$

The benefits of multiple CRMs in a session of treatment for P-SCC-BPPV have been demonstrated in a systematic review, in patients who are not fully cleared of otoconial debris. ${ }^{17}$ In centers where multiple CRMs in a session are performed, a change in the pattern of nystagmus during the execution of multiple Epley's maneuvers (EM) from vertical upbeating torsional to horizontal beating (geotropic or apogeotropic) or to vertical downbeating implies canal switch. The canal switch BPPV is an indication to treat the patient with a different repositioning maneuver. The case presented here initially underwent canal switch from right posterior canalolithiasis to right horizontal canalolithiasis and subsequently the other way around. Such a phenomenon of to-and-fro canal switch in a session of treatment has never been reported hitherto, and the possible causes for its occurrence are discussed in this case report.

Table 1 Demographics of BBPV patients

\begin{tabular}{|l|l|l|l|l|l|}
\hline Authors & No. of patients & P-SCC-BPPV (\%) & H-SCC-BPPV (\%) & A-SCC-BPPV (\%) & Multiple canals (\%) \\
\hline De la Meilleure et al, ${ }^{1} 1996$ & 287 & 78.05 & 16.38 & - & 5.57 \\
\hline Honrubia et al, ${ }^{2} 1999$ & 292 & 85.62 & 5.14 & 1.37 & 7.87 \\
\hline Macias et al, ${ }^{2} 2000$ & 259 & 93.02 & 1.94 & - & 5.04 \\
\hline Korres et al, ${ }^{2} 2002$ & 122 & 90.16 & 3.2 & 1.64 & - \\
\hline Sakaida et al, ${ }^{5} 2003$ & 50 & 56 & 33.33 & - & 6 \\
\hline Imai et al, ${ }^{6} 2005$ & 108 & 64.82 & 11.83 & 1.18 & 1.85 \\
\hline Cakir et al, ${ }^{2} 2006$ & 169 & 85.21 & 11.9 & 21.2 & 1.78 \\
\hline Jackson et al, ${ }^{8} 2007$ & 260 & 66.9 & 35.3 & 2.9 & - \\
\hline Chung et al, ${ }^{9} 2009$ & 589 & 61.8 & & - \\
\hline
\end{tabular}

Abbreviations: A-SCC-BPPV, anterior semicircular canal benign paroxysmal positional vertigo; H-SCC-BPPV, horizontal-semicircular canal benign paroxysmal positional vertigo; P-SCC-BPPV, posterior- semicircular canal benign paroxysmal positional vertigo. 


\section{Case Description}

\section{History}

A 59-year-old female patient presented with a 1-day history of rotatory vertigo as she got out of bed, after awakening from sleep in the morning. There was no history of staggering, diplopia, dysarthria, difficulty in swallowing, hiccups, drooping of upper eyelids, or facial or limb weakness. The neurological examination revealed a normal cranial nerve examination, strength was grade 5/5 in all four limbs with normal deep tendon reflexes, and bilateral plantar reflexes were flexor. There was no history of hypertension, diabetes, coronary artery disease, hypothyroidism, jaundice, craniocervical trauma, cervical radiculopathies, cervical canal stenosis, rheumatoid arthritis, Paget's disease, ankylosing spondylitis, low back dysfunction, spinal cord injuries, and cerebrovascular disease.

\section{Examination}

The general physical examination and vitals of the patient were normal. The screening examination of the cervical spine did not reveal any limitation of movement. The examination of the back region did not reveal kyphoscoliosis. The examination of the lumbosacral spine, including straight-leg raising (SLR) test and reverse SLR test, were normal. The neurological examination revealed normal cranial nerve examination; strength was grade 5/5 in all four limbs with normal deep tendon reflexes, and bilateral plantar reflexes were flexor. The examination of the cerebellar system revealed no spontaneous or gaze-evoked nystagmus, and the author did not observe appendicular or axial incoordination. The otoneurological examination revealed normal vertical and horizontal saccadic and smooth pursuit eye movements. The head impulse test was bilaterally normal. Before carrying out positional tests, spontaneous nystagmus was ruled out by using takeaway Frenzel's goggles. ${ }^{18}$ The Dix -Hallpike test (DHT) was performed with the patient in the long-sitting on the examination table (-Fig. 1). As the patient was very apprehensive, a 4-inch-thick pillow was placed behind her buttocks to be used as a vantage point instead of using the end edge of the examination table during the test. Her head was rotated approximately 45 degrees to first to her left in the yaw axis and she was taken to the supine position so that her head extended 20 degrees as she was laid. As no nystagmus was elicited, after some time she was again made to sit and her head rotated 45 degrees to her right in the yaw axis. Subsequently, she was taken to the supine position again so that her head extended 20 degrees as she was laid. Initially, she closed her eyes but when she opened her eyes an upbeating torsional geotropic positional nystagmus (PN) was observed (-Video 1). The DHT lateralized and localized the disease to the right P-SCC. Supine roll test (SRT) was performed by instructing the patient to be on examination table in the long-sitting position. The distance of her buttocks from the head end of examination table was adjusted so that on taking her to the supine position, the occiput of her head would land on a 4-inch-thick pillow, thus flexing it to approximately 30 degrees. From the sitting position, she was taken to the supine neutral position and maintained in this position for 30 seconds. After this, her head was rotated in the yaw axis first to her right and kept in this position for 15 seconds. Thereafter, the 30-degree flexed head was brought to the supine neutral position and subsequently rotated in the yaw axis to her left and maintained for 15 seconds. There was no lying down nystagmus in the supine neutral position and no PN was elicited on either of the sides. The SRT was interpreted as normal.

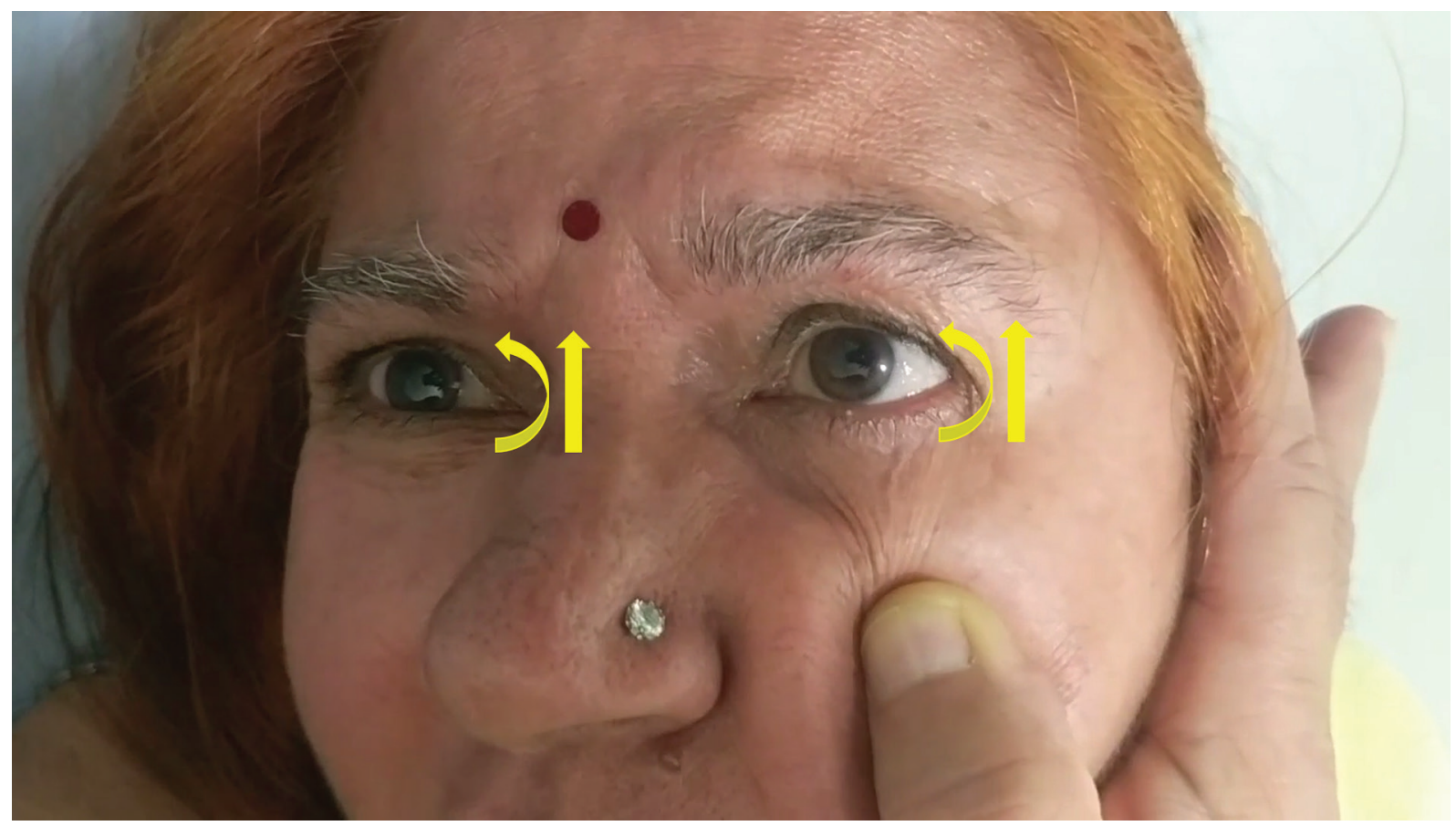

Fig. 1 The Dix-Hallpike test in the right head hanging position elicited an upbeating geotropic (straight arrows) counterclockwise torsional (curved arrows) positional nystagmus, indicating right posterior semicircular canalolithiasis. 


\section{Video 1}

The Dix-Hallpike test in the right head hanging position elicits a counterclockwise torsional (from the examiner's perspective) upbeating positional nystagmus indicating right posterior semicircular canalolithiasis. Online content including video sequences viewable at: https://www.thieme-connect.com/ products/ejournals/html/10.1055/s-0040-1702757.

\section{Diagnosis}

The aforesaid clinical history and examination was consistent with the diagnosis of right posterior semicircular benign paroxysmal positional vertigo (right-P-SCC-BPPV) or right posterior semicircular canalolithiasis.

\section{Intervention}

The patient was treated with consecutive modified right EM, a type of CRM, and postural restrictions between successive maneuvers were not advised initially as per the prevailing recommendations. ${ }^{19}$ The modified right EM (r-MEM) was performed with the patient in the long-sitting position. A 4-inchthick pillow was placed behind her buttocks as a vantage point instead of using the end edge of the treatment table during the r-MEM. ${ }^{20}$ Her head was rotated 45 degrees to the ipsilesional right side in the yaw axis and she was taken to the supine position, so that her head was in 20-degree extension as the cervical rotation was maintained ( - Fig. 2A). During such positioning, upbeating counterclockwise torsional nystagmus was elicited for less than a minute, with the patient experiencing concomitant vertigo for the same duration. Upon completion of the 1 minute with 45-degree cervical rotation to ipsilesional right side, the head was rotated 90 degrees to her left, maintaining its 20-degree extension (-Fig. 2B). Positioning with 45 degrees of cervical rotation to the contralesional left side with the neck in 20-degree extension was maintained for 1 minute; patient neither complained of vertigo nor had any nystagmus during this period. Thereupon, she was instructed to assume the left lateral recumbent position and rotate her head further leftward so that her nose was positioned almost right angled with the treatment table (-Fig. 2C). The latter position was maintained for 1 minute. Thereafter, she was instructed to sequentially dangle down her lower limbs along the free edge of the examination table and while maintaining the head position, she was assisted to assume an upright-sitting position completing one r-MEM ( - Video 5). No vertigo complaint was made by the patient either in the nose-down left-lateral recumbent position or on assuming the upright position after r-MEM completion. However, with the cervical spine in 20-degree extension and 45-degree rotation to the ipsilesional right side (which is equivalent to the right DHT), the patient continued to elicit counterclockwise torsional upbeating PN with concurrent vertigo during second and third successive r-MEMs. During the fourth consecutive r-MEM, apogeotropic horizontal PN without torsional component was observed, as her cervical spine with 45-degree rotation to the ipsilesional right side was brought to the vantage point with the resultant 20 degrees of its extension. Thereupon, the fourth consecutive r-MEM was terminated and a diagnostic SRT was again immediately undertaken. SRT elicited bilateral horizontal apogeotropic PN of more than 1-minute duration that was rather symmetrically strong on either side (-Fig. 3) (-Video 2). It is worth mentioning here that the intensity of the induced nystagmus in patients

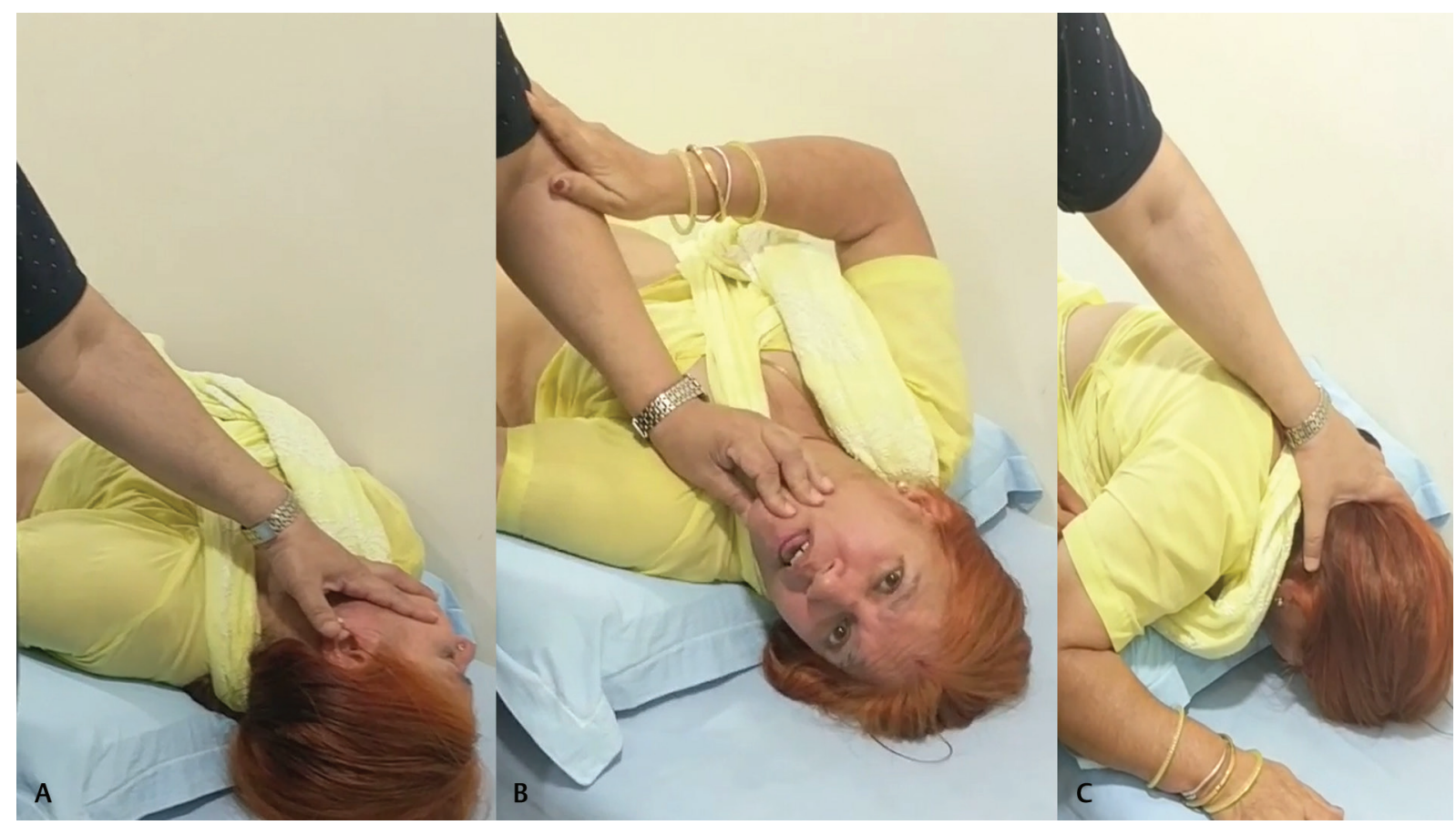

Fig. 2 The three consecutive positions of the modified right Epley's maneuver (r-MEM), using a pillow of 4-inch thickness as the vantage point are (A) ipsilateral cervical rotation in yaw-axis to 45-degrees with 20 degrees of extension, (B) contralateral cervical rotation in yaw-axis to 90-degrees from the position 2A maintaining 20 degrees of extension, and (C) left lateral recumbent position and 45-degrees further contralateral cervical rotation from the position $2 \mathrm{~B}$ so that the nose is at right angle to the plinth of the examination table. 


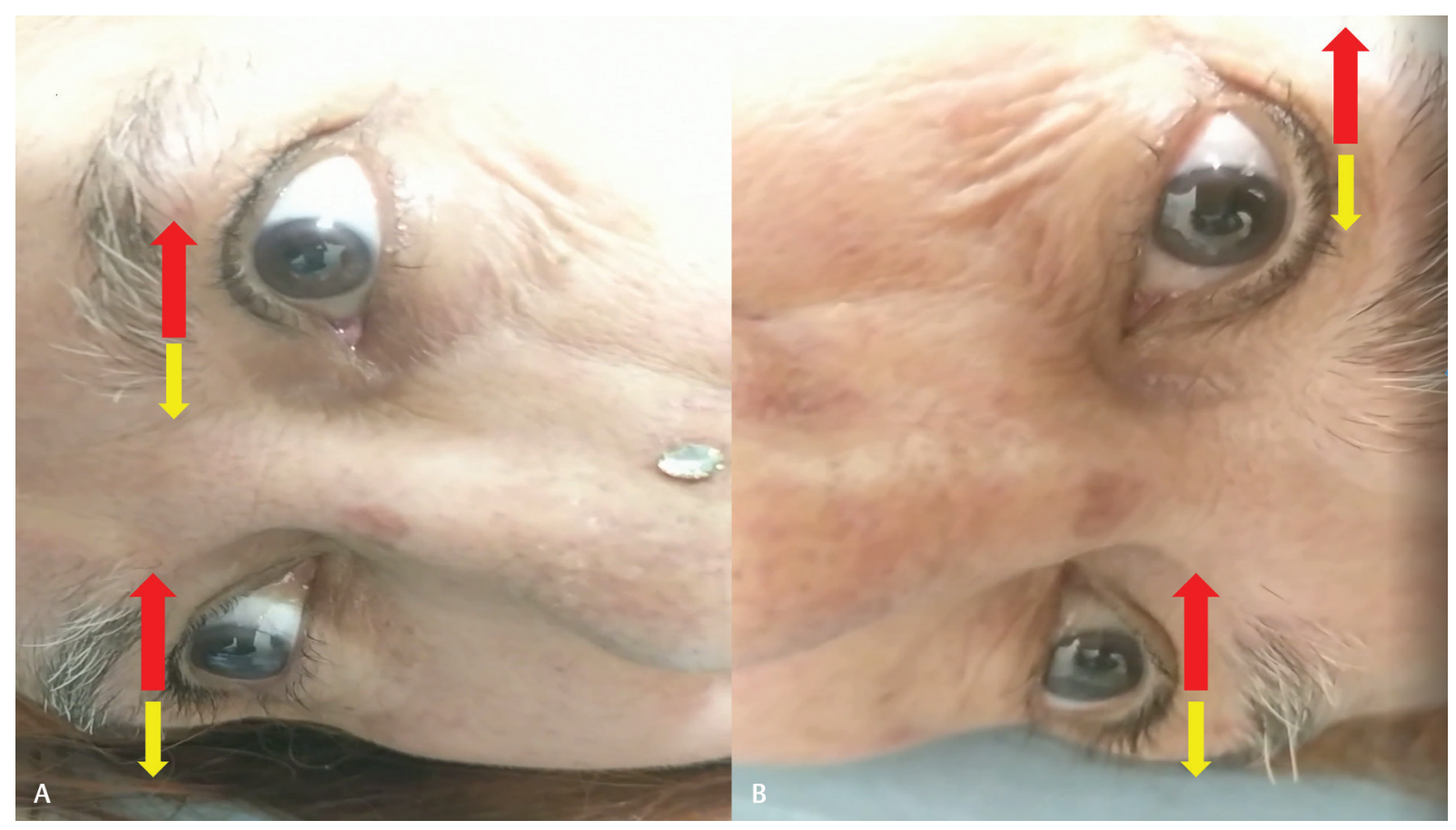

Fig. 3 Supine roll test to the right (A) and to the left (B) elicited apogeotropic horizontal positional nystagmus (red arrow indicates faster component of nystagmus directed away from the ground and yellow arrow indicates slower component directed toward the ground).

\section{Video 2}

The supine roll test is performed with the patient in long sitting on examination table. She is made to lay supine with her head landing on a 4-inch thick pillow resulting in a 30-degree antiflexion of the cervical spine. Thereupon, yawing the head of the patient to the right elicits, after a latency of $4 \mathrm{sec}$, an apogeotropic horizontal positional nystagmus lasting $110 \mathrm{sec}$, and to the left eliciting, after a latency of $28 \mathrm{sec}$, an apogeotropic horizontal positional nystagmus lasting $30 \mathrm{sec}$. The strength of the elicited positional nystagmus is apparently equal to either side. Online content including video sequences viewable at: https://www.thieme-connect.com/products/ejournals/html/ 10.1055/s-0040-1702757.

transiting from P-SCC-BPPV to the H-SCC-BPPV does not always comply well with Ewald's second law, ${ }^{21}$ and for this reason a diagnosis canal switch was considered rather than suspecting polycanalicular BPPV. The diagnosis at this stage of intervention changed from right posterior semicircular canalolithiasis to right short ampullary arm horizontal semicircular canalolithiasis. Treatment with Appiani's maneuver ${ }^{22}$ was initiated and executed twice. The Appiani maneuver was performed by making the patient sit on the couch with the lower limbs dangling down along its long free edge with the patient in short-sitting position ( - Fig. 4A). From this position, she was briskly shifted to right (ipsilesional) lateral recumbent position (-Fig. 4B) and after 1 minute, her head was quickly inclined 45-degree upward in the yaw axis ( $\mathbf{- F i g}$. 4C). The latter position was maintained for 2 minutes after which she was taken to upright-sitting position completing one sequence ( - Video 3 ). An SRT on maximal head positioning to the right in the yaw axis after 1 hour showed counterclockwise torsional (from examiner's perspective) PN (-Fig. 5) (-Video 4), indicating a second canal switch to right posterior semicircular canalolithiasis that was successfully treated again with two sequences of r-MEM ( $\mathbf{- V i d e o ~} 5$ ) done with an interval of 15 minutes apart. Patient was instructed to stay upright for 15 minutes after each maneuver. With the cervical spine in 20-degree extension and 45-degree rotation to the ipsilesional right side (right Dix-Hallpike positioning) during the second consecutive r-MEM, patient did not show any PN and was free of vertigo during and after the maneuver completion. At 24 hours, repeat DHT ( - Video 6). and SRT ( - Video 7 ). were negative and the patient was free of vertigo during and after the positional tests. The seven videos showing changing patterns of PN as the case evolved during diagnostic and therapeutic maneuvers were recorded by the author.

\section{Discussion}

The successful treatment of BPPV with repositioning maneuvers for canalolithiasis has been one of the greatest accomplishments in the field of otoneurology. This has been possible due to several factors such as in vivo demonstration of otoconia in the SCC, application of physical laws to precisely lateralize, as well as localization of the otoconial debris within the SCC, and conceptualization of the movement of otoconia in the SCC during head movements in the physical models of labyrinth to develop specific canal clearing maneuvers. The appearance of a different pattern of PN on a verifying positional test than the one initially observed, after the patient has been subjected to a seemingly successful CRM, is a harbinger of canal switch. A delay of 10 to 15 minutes after CRM is recommended by some 


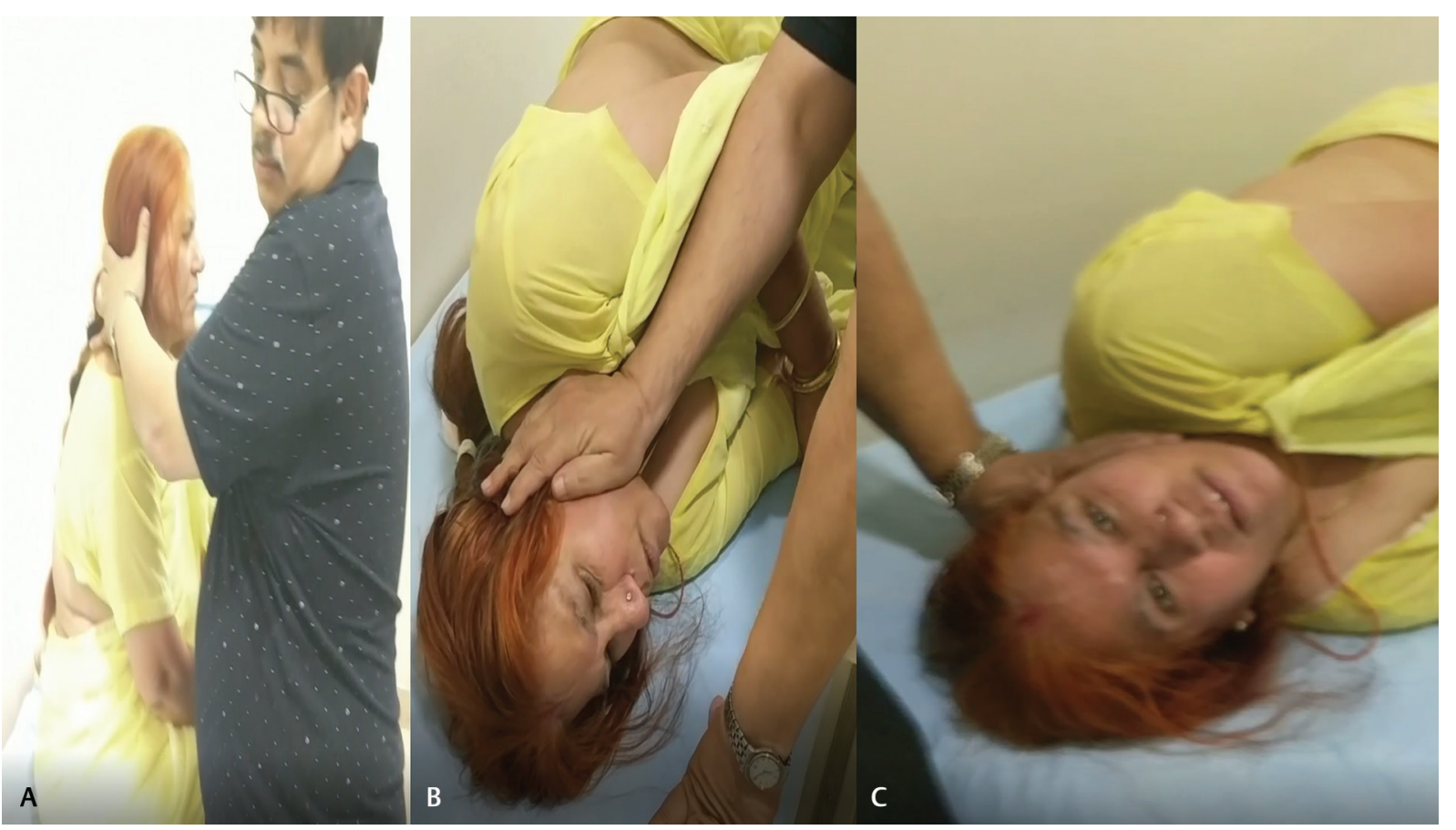

Fig. 4 For Appiani's maneuver, with the patient in short sitting with her lower limbs dangling on the edge of examination table, (A) the patient is moved to the right (ipsilesional) lateral recumbent position (B), after 1 minute her head is quickly inclined 45-degree upward in the yaw axis (C) and maintained for 2 minutes.

\section{Video 3}

Appiani maneuver is performed with the patient in short sitting with her lower limbs hanging down the long edge of examination table. From the upright short sitting, a brisk right (ipsilesional) lateral recumbent positioning is done (step 1), and after a minute, the patient's head is inclined 45-degree upward in the yaw axis and maintained for 2 min (step 2). Thereupon, the patient was positioned upright in the short sitting, completing the Appiani maneuver. Online content including video sequences viewable at: https://www.thieme-connect. com/products/ejournals/html/10.1055/s-0040-1702757.

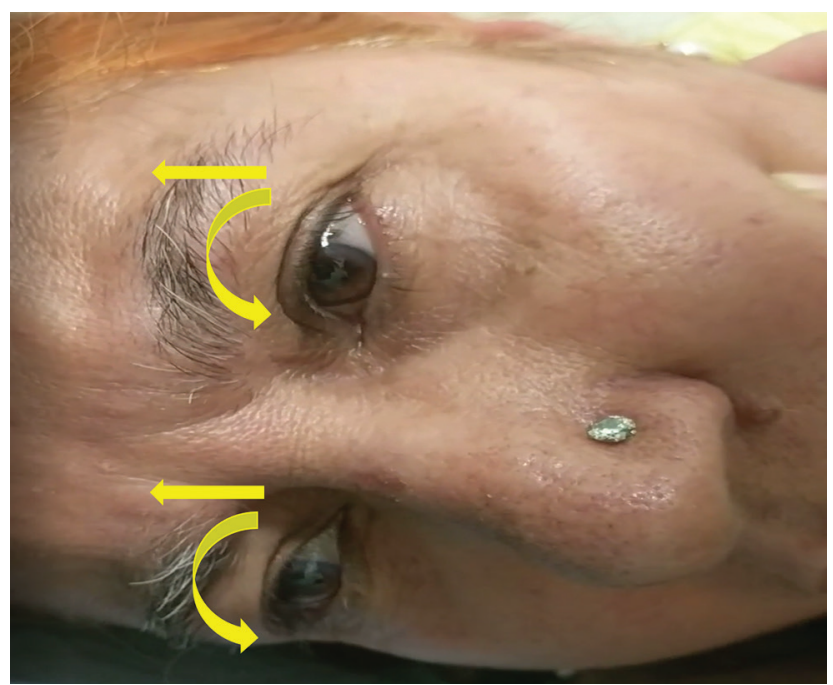

Fig. 5 The supine roll test to the right elicited an upbeating (straight arrows) counterclockwise torsional (curved arrows) positional nystagmus, indicating a second canal switch from right horizontal to the right posterior semicircular canalolithiasis.

\section{Video 4}

The supine roll test is performed with the patient in long sitting on examination table. She is made to lay supine with her head landing on a 4-inch thick pillow resulting in 30-degree antiflexion of the cervical spine. Yawing the head of the patient to the right elicits, after a latency of $2 \mathrm{sec}$, an upbeating counterclockwise torsional (from examiner's perspective) positional nystagmus indicating a second canal-switch of the right short anterior arm horizontal semicircular canalolithiasis to the right posterior semicircular canalolithiasis. Online content including video sequences viewable at: https://www.thieme-connect. com/products/ejournals/html/10.1055/s-0040-1702757.

authors to perform a verifying positional test. . $^{23,24}$ The opening to the horizontal canal is immediately adjacent to the common crus, which increases the risk of canal switch, if verifying positional test is performed earlier. It is speculated that the aggregate of high-mass otoconia are cleared out of canal mostly with lesser number of maneuvers compared with large number of low-mass otoconia dispersed throughout the canal. ${ }^{16}$ The immediate type of canal switch phenomenon is believed to be common in patients cleared with a single maneuver presumably because the otoconia get relocated near the opening to the common crus, at the end of the repositioning maneuver, when patient is taken to the upright position. Restraint in vertical position after CRM for a certain period, plausibly allows the relocated otoconial particles to diffuse away from the common crus area. In patients who undergo multiple EMs in one session of treatment, the position of ipsilesional 45-degree cervical rotation with the neck in 20-degree extension during the consecutive EM simulates the Dix-Hallpike positioning, and verifying positional test as well. Henceforth, successive EM 


\section{Video 5}

Modified right Epley maneuver (m-MEM) is performed with the patient in long sitting on the examination table. A 4-inch thick pillow is placed behind her buttocks as a vantage point instead of using the end edge of the examination table. Her head is rotated 45 degrees to the ipsilesional right side in the yaw axis and she is taken to supine position so that her head is in 20-degree extension as the cervical rotation is maintained. Upon completion of the $1 \mathrm{~min}$ with 45 degrees of cervical rotation to ipsilesional right side, the head is rotated 90 degrees to her left, maintaining its 20-degree extension. Positioning with 45 degrees of cervical rotation to the contralesional left side with the neck in 20 degrees of extension, is maintained for $1 \mathrm{~min}$. Thereupon, she is instructed to assume the left lateral recumbent position and rotate her head further leftward so that her nose is positioned almost right-angled with the examination table. The latter position is maintained for $1 \mathrm{~min}$. Thereupon she is instructed to hang down her lower limbs along the free edge of the examination table and maintaining the head position, she is assisted to assume an upright sitting position completing one r-MEM. Online content including video sequences viewable at: https://www.thieme-connect. com/products/ejournals/html/10.1055/s-0040-1702757.

\section{Video 6}

The Dix-Hallpike test is performed with patient in the long sitting on the examination table such that her right side is toward the long free edge of the table and the distance of her bottoms from the short edge of the table would allow her head to hang when she is laid down. Her head is held with both hands and rotated 45 degrees to her right in the yaw axis. Then, she is made to lay supine in such a manner that her rotated head is extended 20 degrees as she is taken to supine position. This right Dix-Hallpike position is maintained for more than $30 \mathrm{sec}$ and no positional nystagmus is elicited. Online content including video sequences viewable at: https://www.thieme-connect.com/products/ejournals/ html/10.1055/s-0040-1702757.

\section{Video 7}

The supine roll test is performed with the patient in long sitting on examination table such that her right side is toward the long free edge. The distance of her bottoms from the short edge of the table is adjusted so that on taking her to the supine position, the occiput of her head would be placed on the surface of the examination table. From the long sitting, she is taken to supine neutral position and maintained in this position for 30 sec. After this, her head is flexed 30 degrees and then rotated briskly in the yaw axis first to her right and kept in this position for $15 \mathrm{sec}$. Thereafter, the 30 -degree flexed head is brought to the supine neutral position and then rotated in yaw axis briskly to her left and maintained for $15 \mathrm{sec}$. Neither lying-down nystagmus in the supine neutral position nor positional nystagmus is elicited to either of the sides. Online content including video sequences viewable at: https://www.thieme-connect. com/products/ejournals/html/10.1055/s-0040-1702757. performed earlier than the recommended period is also liable to increase the risk of the canal switch for similar reasons. For the H-SCC-BPPV, most of the researchers mention using one to three repositioning maneuvers in one session of treatment and a verifying positional test is performed either immediately ${ }^{22}$ or after 60 minutes of $\mathrm{CRM},{ }^{25-27}$ but there is no recommendation for postural restrictions in these studies.

The documented beneficial effect of multiple CRM in a single session of treatment for the patients with persistent $\mathrm{PN}^{17,19}$ following the initial maneuver, guided us to execute four successive r-MEMs. The case reported here required three r-MEMs in a single session of treatment to effect otoconial movement into the utricle. The patient underwent first immediate type of canal switch from right posterior semicircular canalolithiasis to horizontal semicircular canalolithiasis during fourth successive EM, evident by change of PN pattern from the vertical upbeating to horizontal apogeotropic when the patient was brought to the right Dix-Hallpike position, confirmed by an immediate SRT. This was treated with two sequences of Appiani's maneuver and when patient was subjected 1 hour later to SRT, an upbeating geotropic PN was observed indicating a second immediate type of canal switch from right horizontal semicircular canalolithiasis to right posterior semicircular canalolithiasis. Such a phenomenon of back and forth canal switch in a single session of treatment with CRM has not been reported hitherto. Initially, successive r-MEM was performed without any delay in between the maneuvers during which the first canal switch occurred. After treating the patient with a second sequence of Appiani's maneuver, 1 hour lapsed before a repeat, verifying SRT was performed, revealing an upbeating geotropic PN on right indicating reswitch to right posterior semicircular canalolithiasis. While there is strong recommendation against postural restrictions after CRM for P-SCC-BPPV, there is a distinct lack of recommendation for or against postural restrictions after CRM for H-SCC-BPPV. ${ }^{19}$ Accordingly, no instructions about postural restrictions were given after Appiani's maneuver, the patient during this 1-hour period laid in a left lateral recumbent position on account of fear of having vertigo while lying supine or in the right lateral recumbent position. The second canal switch to right posterior semicircular canalolithiasis was treated with two r-MEMs at an interval of 15 minutes with instructions to stay upright between and after the maneuvers for the same time duration. The patient did not show any PN during Dix-Hallpike position of the second r-MEM and a verifying DHT and SRT done 24 hours later also did not show any PN and the patient was free of vertigo, indicating that patient has recovered completely.

\section{Conclusion}

Canal switch is a well-established clinical entity that needs to be borne in mind by otoneurologist while treating patients with BPPV. An optimal time delay to perform a verifying positional test after therapeutic session with CRM is crucial to prevent the immediate type of reflux of relocated otoconia 
from the utricle into a different semicircular canal. In centers, where more than a single EM is performed in a single session of treatment, a delay of 10 to 15 minutes appears to be appropriate between successive maneuvers. There are speculations that a certain period of restraint in the vertical position after CRM may prevent immediate reflux, but this needs to be confirmed by the randomized control trials.

\section{Note}

The views expressed in the submitted article are author's own and not an official position of the institution to which author is affiliated.

\section{Funding \\ None.}

\section{Conflict of Interest}

None declared.

\section{Acknowledgments}

The author would like to thank Renith Kurian who recorded the video of diagnostic and therapeutic maneuvers and precisely captured the nystagmus during the entire diagnostic and treatment period.

\section{References}

1 De la Meilleure G, Dehaene I, Depondt M, Damman W, Crevits L, Vanhooren G. Benign paroxysmal positional vertigo of the horizontal canal. J Neurol Neurosurg Psychiatry 1996;60(1):68-71

2 Honrubia V, Baloh RW, Harris MR, Jacobson KM. Paroxysmal positional vertigo syndrome. Am J Otol 1999;20(4):465-470

3 Macias JD, Lambert KM, Massingale S, Ellensohn A, Fritz JA. Variables affecting treatment in benign paroxysmal positional vertigo. Laryngoscope 2000;110(11):1921-1924

4 Korres S, Balatsouras DG, Kaberos A, Economou C, Kandiloros D, Ferekidis E. Occurrence of semicircular canal involvement in benign paroxysmal positional vertigo. Otol Neurotol 2002;23(6):926-932

5 Sakaida M, Takeuchi K, Ishinaga H, Adachi M, Majima Y. Longterm outcome of benign paroxysmal positional vertigo. Neurology 2003;60(9):1532-1534

6 Imai T, Ito M, Takeda N, et al. Natural course of the remission of vertigo in patients with benign paroxysmal positional vertigo. Neurology 2005;64(5):920-921

7 Cakir BO, Ercan I, Cakir ZA, Civelek S, Sayin I, Turgut S. What is the true incidence of horizontal semicircular canal benign paroxysmal positional vertigo? Otolaryngol Head Neck Surg 2006;134(3):451-454

8 Jackson LE, Morgan B, Fletcher JC Jr, Krueger WW. Anterior canal benign paroxysmal positional vertigo: an underappreciated entity. Otol Neurotol 2007;28(2):218-222

9 Chung KW, Park KN, Ko MH, et al. Incidence of horizontal canal benign paroxysmal positional vertigo as a function of the duration of symptoms. Otol Neurotol 2009;30(2):202-205

10 von Brevern M, Bertholon P, Brandt $\mathrm{T}$, et al. Benign paroxysmal positional vertigo: Diagnostic criteria. J Vestib Res 2015;25(3-4):105-117
11 Herdman SJ, Tusa RJ. Complications of the canalith repositioning procedure. Arch Otolaryngol Head Neck Surg 1996;122(3):281-286

12 Yimtae K, Srirompotong S, Srirompotong S, Sae-Seaw P. A randomized trial of the canalith repositioning procedure. Laryngoscope 2003;113(5):828-832

13 Anagnostou E, Stamboulis E, Kararizou E. Canal conversion after repositioning procedures: comparison of Semont and Epley maneuver. J Neurol 2014;261(5):866-869

14 Nuti D, Agus G, Barbieri MT, Passali D. The management of horizontal-canal paroxysmal positional vertigo. Acta Otolaryngol 1998;118(4):455-460

15 Park S, Kim BG, Kim SH, Chu H, Song MY, Kim M. Canal conversion between anterior and posterior semicircular canal in benign paroxysmal positional vertigo. Otol Neurotol 2013;34(9):1725-1728

16 Dispenza F, DE Stefano A, Costantino C, et al. Canal switch and re-entry phenomenon in benign paroxysmal positional vertigo: difference between immediate and delayed occurrence. Acta Otorhinolaryngol Ital 2015;35(2):116-120

17 Reinink H, Wegner I, Stegeman I, Grolman W. Rapid systematic review of repeated application of the epley maneuver for treating posterior BPPV. Otolaryngol Head Neck Surg 2014;151(3):399-406

18 Strupp M, Fischer C, Hanß L, Bayer O. The takeaway Frenzel goggles: a Fresnel-based device. Neurology 2014;83(14):1241-1245

19 Bhattacharyya N, Gubbels SP, Schwartz SR, et al. Clinical practice guideline: benign paroxysmal positional vertigo (Update). Otolaryngol Head Neck Surg 2017;156(3_suppl):S1-S47

20 Radtke A, von Brevern M, Tiel-Wilck K, Mainz-Perchalla A, Neuhauser H, Lempert T. Self-treatment of benign paroxysmal positional vertigo: Semont maneuver vs Epley procedure. Neurology 2004;63(1):150-152

21 Koo JW, Moon IJ, Shim WS, Moon SY, Kim JS. Value of lyingdown nystagmus in the lateralization of horizontal semicircular canal benign paroxysmal positional vertigo. Otol Neurotol 2006;27(3):367-371

22 Ciniglio Appiani G, Catania G, Gagliardi M, Cuiuli G. Repositioning maneuver for the treatment of the apogeotropic variant of horizontal canal benign paroxysmal positional vertigo. Otol Neurotol 2005;26(2):257-260

23 Foster CA, Zaccaro K, Strong D. Canal conversion and reentry: a risk of Dix-Hallpike during canalith repositioning procedures. Otol Neurotol 2012;33(2):199-203

24 Oliveira AK, Suzuki FA, Boari L. Is it important to repeat the positioning maneuver after the treatment for benign paroxysmal positional vertigo? Rev Bras Otorrinolaringol (Engl Ed) 2015;81(2):197-201

25 Kim JS, Oh SY, Lee SH, et al. Randomized clinical trial for apogeotropic horizontal canal benign paroxysmal positional vertigo. Neurology 2012;78(3):159-166

26 Mandalà M, Pepponi E, Santoro GP, et al. Double-blind randomized trial on the efficacy of the Gufoni maneuver for treatment of lateral canal BPPV. Laryngoscope 2013;123(7):1782-1786

27 Kim HA, Park SW, Kim J, et al. Efficacy of mastoid oscillation and the Gufoni maneuver for treating apogeotropic horizontal benign positional vertigo: a randomized controlled study. J Neurol 2017;264(5):848-855 